TI 2011-036/1

Tinbergen Institute Discussion Paper

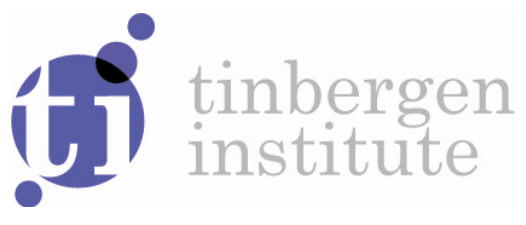

\title{
Games on Union Closed Systems
}

\author{
René van den Brink ${ }^{7}$ \\ I/ya Katsev² \\ Gerard van der Laan'
}

I VU University Amsterdam, and Tinbergen Institute;

${ }^{2}$ Russian Academy of Sciences, St. Petersburg. 
Tinbergen Institute is the graduate school and research institute in economics of Erasmus University Rotterdam, the University of Amsterdam and VU University Amsterdam.

More TI discussion papers can be downloaded at http://www.tinbergen.nl

Tinbergen Institute has two locations:

Tinbergen Institute Amsterdam

Gustav Mahlerplein 117

1082 MS Amsterdam

The Netherlands

Tel.: +31(0)205251600

Tinbergen Institute Rotterdam

Burg. Oudlaan 50

3062 PA Rotterdam

The Netherlands

Tel.: +31(0)10 4088900

Fax: +31(0)104089031

Duisenberg school of finance is a collaboration of the Dutch financial sector and universities, with the ambition to support innovative research and offer top quality academic education in core areas of finance.

DSF research papers can be downloaded at: http://www.dsf.nl/

Duisenberg school of finance

Gustav Mahlerplein 117

1082 MS Amsterdam

The Netherlands

Tel.: +31(0)20 5258579 


\title{
Games on Union Closed Systems ${ }^{1}$
}

\author{
René van den Brink ${ }^{2}$ Ilya Katsev ${ }^{3}$ Gerard van der Laan ${ }^{4}$
}

February 11, 2011

\footnotetext{
${ }^{1}$ This research was partly carried out while the second author was visiting the Tinbergen Institute, VU University Amsterdam, on NWO-grant 047.017.017 within the framework of DutchRussian cooperation.

${ }^{2}$ J.R. van den Brink, Department of Econometrics and Tinbergen Institute, VU University, De Boelelaan 1105, 1081 HV Amsterdam, The Netherlands. E-mail: jrbrink@feweb.vu.nl

${ }^{3}$ I.V. Katsev, St. Petersburg Institute for Economics and Mathematics, Russian Academy of Sciences, Tchaikovsky st. 1, 191187 St. Petersburg. E-mail:katsev@yandex.ru

${ }^{4}$ G. van der Laan, Department of Econometrics and Tinbergen Institute, VU University, De Boelelaan 1105, $1081 \mathrm{HV}$ Amsterdam, The Netherlands. E-mail: glaan@feweb.vu.nl
} 


\begin{abstract}
A situation in which a finite set of players can obtain certain payoffs by cooperation can be described by a cooperative game with transferable utility, or simply a TU-game. A solution for TU-games assigns a set of payoff distributions to every TU-game.

In the literature various models of games with restricted cooperation can be found. So, instead of allowing all subsets of the player set $N$ to form, it is assumed that the set of feasible coalitions is a subset of the power set of $N$. In this paper we consider such sets of feasible coalitions that are closed under union, i.e. for any two feasible coalitions also their union is feasible. Properties of solutions (the core, the nucleolus, the prekernel and the Shapley value) are given for games on union closed systems.
\end{abstract}

Keywords: TU-game, restricted cooperation, union closed system, core, prekernel, nucleolus.

AMS subject classification: $91 \mathrm{~A} 12,5 \mathrm{C} 20$

JEL code: C71 


\section{Introduction}

A cooperative game with transferable utility, or simply a TU-game, is a finite set of players and for any subset (coalition) of players a worth representing the total payoff that the coalition can obtain by cooperating. A (single-valued) solution is a function that assigns to every game a payoff vector which components are the individual payoffs of the players.

In its classical interpretation, a TU-game describes a situation in which the players in every coalition $S$ of $N$ can cooperate to form a feasible coalition and earn its worth. In the literature various restrictions on coalition formation are developed. ${ }^{1}$ For example, in the (communication) graph games of Myerson (1977) a coalition is feasible if it is connected in a given (communication) graph. Games in which the collection of feasible coalitions forms an antimatroid ${ }^{2}$ are considered in Algaba, Bilbao, van den Brink and JiménezLosada (2003, 2004). A well-known example of an antimatroid is the collection of feasible coalitions induced by a acyclic permission structure, i.e. players need permission from (some of) their superiors in a hierarchical structure when they want cooperate with others. Games with a permission structure are considered in e.g. Gilles, Owen and van den Brink (1992), van den Brink and Gilles (1996), Gilles and Owen (1994) and van den Brink (1997). A model that generalizes both the communication graph games as well as the games on antimatroids are the games on augmenting systems, see Bilbao (2003), Bilbao and Ordóñez (2009) and Algaba, Bilbao and Slikker (2010).

In this paper we consider games with restricted cooperation given by a collection of feasible coalitions that is closed under union, meaning that for any pair of feasible coalitions also their union is feasible. Since such collections are more general than antimatroids, the class of games on union closed systems contains the class of games on antimatroids. In van den Brink, Katsev and van der Laan (2010) two single-valued solutions for games on union closed systems that generalize the Shapley value are defined and characterized. The first solution is based on games with a permission structure, the other directly applies the Shapley value to some restricted game. Both solutions generalize the Shapley value in the sense that they are equal to the Shapley value when the union closed system is the power set of player set $N$.

The restricted game considered in van den Brink et al. (2010) is defined by assigning to each coalition the worth of its maximal feasible subset in the union closed system. In this paper we apply several well-known solution concepts as the core, nucleolus, prekernel and Shapley value to this restricted game. We show some interesting properties of these

\footnotetext{
${ }^{1}$ For a survey on we refer to Bilbao (2000).

${ }^{2} \mathrm{~A}$ collection of feasible coalitions $\mathcal{A} \subseteq 2^{N}$ is an antimatroid if it (i) contains the $\emptyset$, (ii) is union closed (when $S, T \in \mathcal{A}$, then also $S \cup T \in \mathcal{A}$ ), and (iii) satisfies accessibility (for every $S \in \mathcal{A}$ there is $i \in S$ such that $S \backslash\{i\} \in \mathcal{A}$ ), see Dilworth (1940) and Edelman and Jamison (1985).
} 
solutions on the class of games on union closed systems, in particular for monotone games. We also give a sufficient condition to guarantee that the nucleolus is the unique point in the intersection of the prekernel and the core.

This paper is organized as follows. Section 2 is a preliminary section on cooperative TU-games. In Section 3 we introduce games on union closed systems. Section 4 discusses properties of some solutions for monotone games on union closed systems. Finally, Section 5 gives special attention to the prekernel.

\section{TU-games and solutions}

A situation in which a finite set of players can obtain certain payoffs by cooperating can be described by a cooperative game with transferable utility, or simply a TU-game, being a pair $(N, v)$, where $N \subset \mathbb{N}$ is a finite set of $n$ players and $v: 2^{N} \rightarrow \mathbb{R}$ is a characteristic function on $N$ such that $v(\emptyset)=0$. For any coalition $S \subseteq N, v(S)$ is the worth of coalition $S$, i.e., the members of coalition $S$ can obtain a total payoff of $v(S)$ by agreeing to cooperate. For ease of notation we write $v(i)=v(\{i\})$ for $i \in N$. A player $i \in N$ is called a veto player if $v(S)=0$ if $i \notin S$ and a game $v$ is veto-rich if it contains at least one veto player. Since we take the player set $N$ to be fixed, we denote the game $(N, v)$ just by its characteristic function $v$. For each nonempty $T \subseteq N$, the unanimity game $u_{T}$ is given by $u_{T}(S)=1$ if $T \subseteq S$, and $u_{T}(S)=0$ otherwise. It is well-known that the unanimity games form a basis for $\mathcal{G}^{N}$. For every $v \in \mathcal{G}^{N}$ it holds that $v=\sum_{T \subset N, T \neq \emptyset} \Delta_{T}(v) u_{T}$, where $\Delta_{T}(v)=\sum_{S \subseteq T}(-1)^{|T|-|S|} v(S)$ are the Harsanyi dividends, see Harsanyi (1959).

We denote the collection of all characteristic functions on $N$ by $\mathcal{G}^{N}$ and $n=|N|$ denotes the cardinality of $N$. A game $v \in \mathcal{G}^{N}$ is monotone if $v(S) \leq v(T)$ for all $S \subseteq T \subseteq N$. We denote by $\mathcal{G}_{m}^{N}$ the class of all monotone TU-games on $N$.

A payoff vector is a vector $x \in \mathbb{R}^{n}$ assigning a payoff $x_{i}$ to every $i \in N$. In the sequel, for $S \subseteq N$ we denote $x(S)=\sum_{i \in S} x_{i}$. The set of efficient payoff vectors of a game $v \in \mathcal{G}^{N}$ is given by

$$
X(v)=\left\{x \in \mathbb{R}^{n} \mid x(N)=v(N)\right\}
$$

and the imputation set is the set of efficient and individually rational payoff vectors given by

$$
I(v)=\left\{x \in X(v) \mid x_{i} \geq v(i) \text { for every } i \in N\right\} .
$$

A (set-valued) solution is a mapping $F: \mathcal{G}^{N} \rightarrow \mathbb{R}^{n}$ that assigns a (possibly empty) set $F(v) \subset \mathbb{R}^{n}$ of payoff vectors to every $v \in \mathcal{G}^{N}$. A solution $F$ is said to be single-valued if it assigns to every $v \in \mathcal{G}^{N}$ a single payoff vector $F(v) \in \mathbb{R}^{n}$. Notice that $F=X$ and $F=I$ 
are set-valued solutions assigning to every $v$ the set of efficient payoff vectors, respectively the imputation set. The most well-known set-valued solution is the core $C$, assigning to every $v \in \mathcal{G}^{N}$ the set

$$
C(v)=\{x \in X(v) \mid x(S) \geq v(S) \text { for every } S \subset N\} .
$$

Since $I(v) \subseteq C(v)$, the core is the set of stable imputations in the sense that no coalition $S$ can improve by separating from the grand coalition $N$.

A collection $\mathcal{B}$ of subsets $B$ of $N$ is said to be a balanced collection when the system of equations

$$
\sum_{B \in \mathcal{B}} \lambda_{B} e^{B}=e^{N}
$$

has a positive solution, where for any $B \subseteq N$ the vector $e^{B} \in \mathbb{R}^{n}$ is defined by $e_{j}^{B}=1$ when $j \in B$ and $e_{j}^{B}=0$ otherwise. A game $v \in \mathcal{G}^{N}$ is balanced if

$$
\sum_{j=1}^{m} \lambda_{j}^{B} v\left(S_{j}\right) \leq v(N)
$$

for every balanced collection $\mathcal{B}=\left\{S_{1}, \ldots, S_{m}\right\} \in \mathcal{B}$. A well-known result states that the core of a game is non-empty if and only if the game is balanced, see Bondareva (1962) or Shapley (1967). Notice that every veto-rich monotone game has a non-empty core (any payoff vector that assigns worth $v(N)$ to the veto players is in the core) and thus is balanced.

Two other well-known solutions are the (pre)nucleolus and the (pre)kernel. To define the (pre)nucleolus of a game $v \in \mathcal{G}^{N}$, let $x \in \mathbb{R}^{n}$ be a payoff vector. Then the excess $e(S, x)$ of coalition $S \subseteq N$ is defined by

$$
e(S, x)=v(S)-x(S)
$$

Further, let $E(x)$ be the $\left(2^{n}-2\right)$-component vector that is composed of the excesses of all coalitions $S \subset N, S \neq \emptyset$, in a non-increasing order, so $E_{1}(x) \geq E_{2}(x) \geq \ldots \geq E_{2^{n}-2}(x)$. Then the prenucleolus $P N(v)$ of a game $v \in \mathcal{G}^{N}$ is the unique efficient payoff vector which lexicographically minimizes the vector-valued function $E(\cdot)$ over the set of efficient payoff vectors. Formally,

$$
P N(v)=x \text { such that } x \in X(v) \text { and } E(x) \preceq_{L} E(y) \text { for all } y \in X(v) \text {, }
$$

where $\preceq_{L}$ denotes the lexicographic order of vectors. The nucleolus $N u c(v)$ of a game $v \in \mathcal{G}^{N}$ is the unique imputation which lexicographically minimizes the vector-valued function $E(\cdot)$ over the imputation set, so

$$
N u c(v)=x \text { such that } x \in I(v) \text { and } E(x) \preceq_{L} E(y) \text { for all } y \in I(v) \text {. }
$$


Both the prenucleolus and the nucleolus are single-valued solutions.

To define the prekernel and the kernel of a game $v \in \mathcal{G}^{N}$ we first introduce the notion of complaint. For a payoff vector $x \in \mathbb{R}^{n}$, the complaint of player $i \in N$ against another player $j \in N$ is given by

$$
s_{i j}(x)=\max _{\{S \subseteq N \mid i \in S, j \notin S\}}(v(S)-x(S)) .
$$

The prekernel $P K$ assigns to every $v \in \mathcal{G}^{N}$ the set of efficient payoff vectors

$$
P K(v)=\left\{x \in X(v) \mid s_{i j}(x)=s_{j i}(x) \text { for all } i, j \in N\right\}
$$

and the kernel $K$ assigns to every $v \in \mathcal{G}^{N}$ the set of imputations

$$
K(v)=\left\{x \in I(v) \mid\left[s_{i j}(x)=s_{j i}(x)\right] \text { or }\left[s_{i j}(x)>s_{j i}(x) \text { and } x_{j}=v(j)\right] \text { for all } i, j \in N\right\} .
$$

Finally we define the least core $L C(v)$ of a game $v \in \mathcal{G}^{N}$. For an efficient payoff vector $x \in X(v)$, the excess $e_{v}(x)$ of $x$ is defined by

$$
e_{v}(x)=\max _{\left\{S \in 2^{N} \mid S \neq \emptyset, N\right\}} e(S, x)=\max _{\left\{S \in 2^{N} \mid S \neq \emptyset, N\right\}}(v(S)-x(S)),
$$

i.e. for any coalition $S \neq \emptyset, N$, its payoff $x(S)$ is at least equal to its own worth $v(S)$ minus the excess $e_{v}(x)$ with equality for at least one of these coalitions. Further the gain $e(v)$ of $v$ is defined as the largest negative excess, thus

$$
e(v)=\max _{x \in X(N, v)}-e_{v}(x) .
$$

Notice that $e_{v}(x) \leq 0$ when $x \in C(v)$ and $e(v) \geq 0$ if and only if $C(v) \neq \emptyset$. Then the least core, introduced by Maschler, Peleg and Shapley (1979), see e.g. also Einy, Holzman and Monderer (1999), is defined as the solution $L C$ that assigns to game $v$ the set of efficient payoff vectors

$$
L C(v)=\{x \in X(v) \mid x(S) \geq v(S)+e(v) \text { for every } S \neq \emptyset, N\} .
$$

Observe that $L C(v) \subseteq C(v)$ if $C(v) \neq \emptyset$, with $L C(v)=C(v)$ when $e(v)=0$. We also have that $N u c(v) \in L C(v)$ and that $L C(v) \subseteq I(v)$ when $v \in \mathcal{G}_{m}^{N}$.

\section{Games on union closed systems}

We now consider tuples $(v, \Omega)$, where $v$ is a TU-game on player set $N$ and $\Omega \subseteq 2^{N}$ is a collection of subsets of $N$. We call such a tuple a game with restricted cooperation. In such a game the collection of subsets $\Omega$ restricts the cooperation possibilities of the players in $N$. We say that a coalition $S \in 2^{N}$ is feasible if and only if $S \in \Omega$. In this paper we only consider sets of feasible coalitions that are closed under union. 
Definition 3.1 A collection $\Omega \subseteq 2^{N}$ is a union closed system of coalitions if

1. $\emptyset, N \in \Omega$,

2. If $S, T \in \Omega$, then $S \cup T \in \Omega$.

In the sequel we denote the collection of all union closed systems in $2^{N}$ by $\mathcal{C}^{N}$.

\section{Example 3.2}

1. Both $\Omega=\{\emptyset, N\}$ and $\Omega=2^{N}$ are union closed systems, the first one is the smallest union closed system and the second one is the largest union closed system of subsets of $N$, i.e. $\{\emptyset, N\} \subseteq \Omega \subseteq 2^{N}$ for every union closed system $\Omega$ of subsets of $N$.

2. For some $k \in\{1, \ldots|N|\}$, the collection of coalitions $\Omega=\{S \subseteq N|| S \mid \geq k\} \cup\{\emptyset\}$ is closed under union. More generally, let the collection $\mathcal{P}=\left\{P^{1}, \ldots, P^{m}\right\}$ of nonempty subsets of $N$ be a partition of $N$, and for every $P^{k}, k \in\{1, \ldots, m\}$, let $q_{k} \in\left\{1, \ldots,\left|P^{k}\right|\right\}$ be a quotum meaning that a nonempty coaliton $S \subseteq N$ can form $S$ contains at least $q_{k}$ players from $P^{k}$ for every $k=1, \ldots, m$. The collection of feasible coalitions

$$
\Omega=\left\{S \subseteq N|| S \cap P_{k} \mid \geq q_{k} \text { for all } k \in\{1, \ldots, m\}\right\} \cup\{\emptyset\}
$$

is closed under union.

3. Let $D$ be an acyclic directed graph on player set $N$ (representing for instance some hierarchical structure), and let $\Omega$ be the collection of subsets of $N$ such that $S \in \Omega$ whenever for every $i \in S$ also all predecessors of $i$ in the digraph $D$ belong to $S$. Then $\Omega$ is union closed. Also the collection $\Omega$ of subsets of $N$ such that $S \in \Omega$ whenever for every $i \in N$ having a predecesssor in $D$ at least one of the predecessors is in $S$ is union closed. For given $D$ these collections are called the collection of conjunctive, respectively disjunctive, feasible coalitions and are an antimatroid, see Algaba et al. (2003). Every antimatroid is a union closed system by definition ${ }^{3}$.

For notational convenience we require in Definition 3.1 that the grand coalition $N$ is feasible. The results in this paper can be modified to hold without this requirement if in the axioms we distinguish between players that belong to at least one feasible coalition and those that do not belong to any feasible coalition. Note that union closedness implies that the grand coalition is feasible if every player belongs to at least one feasible coalition. So, instead of assuming that $N \in \Omega$ we could do with the weaker normality assumption

\footnotetext{
${ }^{3}$ In fact, an augmenting system is an antimatroid if and only if it is closed under union, see Bilbao (2003) and Algaba, Bilbao and Slikker (2010). Note that examples 1 and 2 above are union closed systems that are not antimatroids.
} 
stating that every player belongs to at least one feasible coalition. We give some definitions and properties for union closed systems.

Definition 3.3 For two players $i, j \in N, i \neq j$, player $i$ is a superior of player $j$ in $\Omega \in \mathcal{C}^{N}$, if $i \in S$ for every $S \in \Omega$ such that $j \in S$. In that case player $j$ is a subordinate of $i$.

Corollary 3.4 If $i$ is a superior of $j$ in $\Omega$ and $k$ is a superior of $i$ in $\Omega$ then $k$ is a superior of $j$ in $\Omega$.

Further, for $i \in N$ and $\Omega \in \mathcal{C}^{N}$ define

$S_{i}^{\Omega}=\{j \in N \mid j=i$ or $i$ is a superior of $j\}$,

i.e. $S_{i}^{\Omega} \subseteq N$ denotes the set containing player $i$ and all subordinates of $i$ in $\Omega$. Then the next proposition says that when $\Omega$ is a union closed system, for every $i \in N$ the complement of $i$ and all its subordinates is in $\Omega$.

Proposition 3.5 When $\Omega \in \mathcal{C}^{N}$, then $N \backslash S_{i}^{\Omega} \in \Omega$ for every $i \in N$.

Proof. Let $U$ be the union of all feasible sets not containing $i$. Since $\Omega \in \mathcal{C}^{N}$, it follows that $U \in \Omega$. Further, by definition of $U$ we have that $i \notin U$. Consider a player $j \notin U$ with $j \neq i$. It holds that any feasible set without $i$ does not contain $j$. So $i$ is a superior of $j$ and thus $j \in S_{i}^{\Omega}$. Hence $N \backslash U \subseteq S_{i}^{\Omega}$. On the other hand, consider some player $j \in S_{i}^{\Omega}$. If $j=i$ then $j \notin U$ by definition of $U$. If $j \neq i$, then any feasible set containing $j$ also contains $i$. Hence $j \notin U$, which shows that $S_{i}^{\Omega} \subseteq N \backslash U$. Hence $N \backslash S_{i}^{\Omega}=U \in \Omega$.

A set $S \subseteq N$ of players can attain its value $v(S)$ if $S \in \Omega$. When $S \notin \Omega$ then coalition $S$ can not be formed and so the set $S$ of players can not realize its worth $v(S)$. For a tuple $(v, \Omega)$, let $\sigma_{\Omega}: 2^{N} \rightarrow \Omega$ be given by $\sigma_{\Omega}(S)=\cup_{\{E \in \Omega \mid E \subseteq S\}} E$, i.e. $\sigma_{\Omega}(S)$ is the largest feasible subset of $S$ in the system $\Omega$. By union closedness this largest feasible subset is unique. We then define the restricted game $r_{v, \Omega} \in \mathcal{G}^{N}$ of $(v, \Omega)$ by

$$
r_{v, \Omega}(S)=v\left(\sigma_{\Omega}(S)\right)
$$

i.e. the restricted game is a standard TU-game that assigns to each coalition $S \subseteq N$ the worth of its largest feasible subset. Notice that when $\Omega=\{\emptyset, N\}$, then $\sigma_{\Omega}(N)=N$ and $\sigma_{\Omega}(S)=\emptyset$ for all $S \neq N$ and thus $r_{v, \Omega}(N)=v(N)$ and $r_{v, \Omega}(S)=v(\emptyset)=0$ for every $S \neq N$. Thus the restricted game $r_{v, \Omega}$ is a multiple of the unanimity game of $N$, being a game in which every player is a veto-player. When $\Omega=2^{N}$ then $\sigma_{\Omega}(S)=S$ and $r_{v, \Omega}(S)=v(S)$ for every $S \subseteq N$. In this case the restricted game $r_{v, \Omega}$ coincides with $v$.

Next we generalize some inheritance properties of the restricted game which generalizes known results for games with a permission tructure and games on antimatroids. 
Proposition 3.6 Let $\Omega \in \mathcal{C}^{N}$ and let $v \in \mathcal{G}^{N}$ be a monotone game. Then

1. the restricted game $r_{v, \Omega}$ is monotone;

2. if $v$ is superadditive then $r_{v, \Omega}$ is superadditive;

3. if $v$ is balanced then $r_{v, \Omega}$ is balanced;

4. if $v$ is convex and $\Omega$ is closed under intersection (i.e. $S, T \in \Omega$ implies that $S \cap T \in$ $\Omega)$, then $r_{v, \Omega}$ is convex.

Proof. Let $\Omega \in \mathcal{C}^{N}$ and let $v \in \mathcal{G}^{N}$ be a monotone game.

1. By definition of $\sigma_{\Omega}$ it is obvious that $S \subseteq T$ implies that $\sigma_{\Omega}(S) \subseteq \sigma_{\Omega}(T)$, and thus by monotonicity of $v, S \subseteq T$ implies that $r_{v, \Omega}(S)=v\left(\sigma_{\Omega}(S)\right) \leq v\left(\sigma_{\Omega}(T)\right)=r_{v, \Omega}(T)$, showing monotonicity of $r_{v, \Omega}$.

2. By union closedness, $\sigma_{\Omega}(S) \cup \sigma_{\Omega}(T) \in \Omega$ for all $S, T \in \Omega$. Since $\sigma_{\Omega}(S) \cup \sigma_{\Omega}(T) \subseteq S \cup T$, we then have $\sigma_{\Omega}(S) \cup \sigma_{\Omega}(T) \subseteq \sigma_{\Omega}(S \cup T)$. If $S \cap T=\emptyset$ then $\sigma_{\Omega}(S) \cap \sigma_{\Omega}(T)=\emptyset$, and thus $r_{v, \Omega}(S)+r_{v, \Omega}(T)=v\left(\sigma_{\Omega}(S)\right)+v\left(\sigma_{\Omega}(T)\right) \leq v\left(\sigma_{\Omega}(S) \cup \sigma_{\Omega}(T)\right) \leq v\left(\sigma_{\Omega}(S \cup T)\right)=$ $r_{v, \Omega}(S \cup T)$, where the first inequality follows from superadditivity of $v$. This shows superadditivity of $r_{v, \Omega}$.

3. This follows from Proposition 4.1 and the obvious fact that $C(v) \subseteq C^{*}(v, \Omega)$.

4. In 2 we already showed that by union closedness $\sigma_{\Omega}(S) \cup \sigma_{\Omega}(T) \subseteq \sigma_{\Omega}(S \cup T)$. Similar, by intersection closedness $\sigma_{\Omega}(S) \cap \sigma_{\Omega}(T) \in \Omega$ for all $S, T \in \Omega$. Since $\sigma_{\Omega}(S) \cap \sigma_{\Omega}(T) \subseteq$ $S \cap T$, we have $\sigma_{\Omega}(S) \cap \sigma_{\Omega}(T) \subseteq \sigma_{\Omega}(S \cap T)$. But then $r_{v, \Omega}(S)+r_{v, \Omega}(T)=v\left(\sigma_{\Omega}(S)\right)+$ $v\left(\sigma_{\Omega}(T)\right) \leq v\left(\sigma_{\Omega}(S) \cup \sigma_{\Omega}(T)\right)+v\left(\sigma_{\Omega}(S) \cap \sigma_{\Omega}(T)\right) \leq v\left(\sigma_{\Omega}(S \cup T)\right)+v\left(\sigma_{\Omega}(S \cap T)\right)=$ $r_{v, \Omega}(S \cup T)+r_{v, \Omega}(S \cap T)$, where the first inequality follows from convexity of $v$. This shows convexity of $r_{v, \Omega}$.

A solution for games on union closed systems is a mapping $F$ that assigns a set of payoff vectors $F(v, \Omega) \subset \mathbb{R}^{n}$ to every $v \in \mathcal{G}^{N}$ and $\Omega \in \mathcal{C}^{N}$. In this paper we only consider solutions for games on union closed systems that assign to each tuple $(v, \Omega) \in \mathcal{G}^{N} \times \mathcal{C}^{N}$ the set of payoff vectors $F\left(r_{v, \Omega}\right)$ of a solution $F: \mathcal{G}^{N} \rightarrow \mathbb{R}^{n}$, i.e. a solution for games on a union closed system assigns the set of payoff vectors that is assigned by a solution $F$ on $\mathcal{G}^{N}$ to the restricted game $r_{v, \Omega}$. For ease of notation we denote $F(v, \Omega)=F\left(r_{v, \Omega}\right)$. 


\section{Properties of solutions for the class of monotone games on union closed systems}

In this section we apply the solutions for TU games given in Section 2 and consider their properties for games on union closed systems, in particular we consider the relation between the payoffs of some player $j$ and its superior $i$ for monotone games on union closed systems. Notice that when $v$ is monotone, it holds that for every $\Omega \in \mathcal{C}^{N}$ also the restricted game $r_{v, \Omega}$ is monotone (by Proposition 3.6). Further it should be noticed that $r_{v, \Omega}(\{j\})=v(\emptyset)=0$ when $j$ has a superior, because $\{j\}$ is not feasible when $j$ has a superior.

First we consider the core and the least core of the restricted game. When we take as solution $F$ the core of a game, then we obtain

$$
C(v, \Omega)=C\left(r_{v, \Omega}\right)=\left\{x \in X\left(r_{v, \Omega}\right) \mid x(S) \geq v\left(\sigma_{\Omega}(S)\right), S \subset N\right\}
$$

For a tuple $(v, \Omega)$, let $C^{*}(v, \Omega)$ be given by

$$
C^{*}(v, \Omega)=\left\{x \in X(v) \mid x(S) \geq v(S) \text { for any } S \in \Omega \text { and } x_{j} \geq 0 \text { for any } j \in N\right\} .
$$

i.e. $C^{*}(v, \Omega)$ is the set of nonnegative efficient payoff vectors satisfying the core inequalities corresponding to the feasible coalitions in $\Omega$. It turns out that this set is equal to the core on the class of monotone games on union closed systems.

Proposition 4.1 For every $v \in \mathcal{G}_{m}^{N}$ and $\Omega \in \mathcal{C}^{N}$ we have $C(v, \Omega)=C^{*}(v, \Omega)$.

Proof. Since $N \in \Omega$ we have that $\sigma_{\Omega}(N)=N$ and thus $v\left(\sigma_{\Omega}(N)\right)=v(N)$ and $X\left(r_{v, \Omega}\right)=$ $X(v)$. Let $x \in C(v, \Omega)$. When the singleton player set $\{j\} \in \Omega$, then $\sigma_{\Omega}(\{j\})=\{j\}$ and thus $v\left(\sigma_{\Omega}(\{j\})\right)=v(\{j\}) \geq 0$, since $v \in \mathcal{G}_{m}^{N}$. Otherwise $\sigma_{\Omega}(\{j\})=\emptyset$ and thus $v\left(\sigma_{\Omega}(\{j\})\right)=v(\emptyset)=0$. Hence for every $x \in C(v, \Omega)$ we have that $x_{j} \geq v\left(\sigma_{\Omega}(\{j\})\right) \geq 0$ for every $j \in N$. Further, since $\sigma_{\Omega}(S)=S$ if $S \in \Omega$, the inequalities $x(S) \geq v\left(\sigma_{\Omega}(S)\right)$ for every $S \subset N$, imply that $x(S) \geq v(S)$ for every $S \in \Omega$. Thus, $x \in C^{*}(v, \Omega)$.

Next, let $x \in C^{*}(v, \Omega)$. Obviously, $x(S) \geq v\left(\sigma_{\Omega}(S)\right)$ for every $S \in \Omega$. Since $\sigma_{\Omega}(S) \subseteq S$ and $x_{j} \geq 0$ for all $j \in N$, we have for any $S \subset N$ and $v \in \mathcal{G}_{m}^{N}$ that $x(S) \geq$ $x\left(\sigma_{\Omega}(S)\right) \geq v\left(\sigma_{\Omega}(S)\right)$ for every $S \subset N$. Thus, $x \in C(v, \Omega)$.

Next recall that the least core of a monotone game is contained in the imputation set of the game. Since the restricted game of a monotone game is also monotone, it follows for $v \in \mathcal{G}_{m}^{N}$ that $x \in I\left(r_{v, \Omega}\right)$ for every $x \in L C\left(r_{v, \Omega}\right)$. Since, for every $j \in N$, either $\{j\}$ is feasible in $\Omega$ and thus $x_{j} \geq v(\{j\}) \geq 0$, or $j$ is not feasible and $x_{j} \geq r_{v, \Omega}(\{j\})=v(\emptyset)=0$, we have the following proposition. 
Proposition 4.2 Let $v \in \mathcal{G}_{m}^{N}$ be monotone, and $\Omega \in \mathcal{C}^{N}$. Then $x_{j} \geq 0$ for every $x \in$ $L C\left(r_{v, \Omega}\right)$ and $j \in N$.

For a monotone game $v$ it is straightforward that for two union closed systems $\Omega_{1}$ and $\Omega_{2}$ such that $\Omega_{1} \subseteq \Omega_{2}$, we have $r_{v, \Omega_{1}}(S) \leq r_{v, \Omega_{2}}(S)$ for every $S \in 2^{N}$. Therefore the next proposition follows immediately without proof.

Proposition 4.3 Let $v$ be monotone and $\Omega_{1}, \Omega_{2}$ be two union closed systems such that $\Omega_{1} \subseteq \Omega_{2}$. Then $C\left(v, \Omega_{2}\right) \subseteq C\left(v, \Omega_{1}\right)$.

Since $r_{v, \Omega}=v$, and thus $C(v, \Omega)=C(v)$ when $\Omega=2^{N}$, Proposition 4.3 yields that $C(v, \Omega) \neq \emptyset$ for any $\Omega \in \mathcal{C}^{N}$ and $v \in \mathcal{G}_{m}^{N}$ with non-empty core.

In the following, let $i$ and $j$ be two fixed players such that $i$ is a superior of $j$ in $\Omega$ (and thus $r_{v, \Omega}(j)=0$ ). For a vector $x$ with $x_{j}>0$ and some number $0 \leq a \leq x_{j}$, we denote for fixed $i$ and $j$ the vector $x^{a}$ by $^{4}$

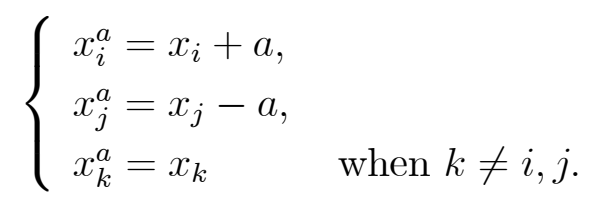

Clearly, since $x_{j}^{a}=x_{j}-a \geq 0=r_{v, \Omega}(\{j\})$ we have that $x^{a} \in I\left(N, r_{v, \Omega}\right)$ when $x \in I\left(N, r_{v, \Omega}\right)$. Moreover, for $S \subset N$

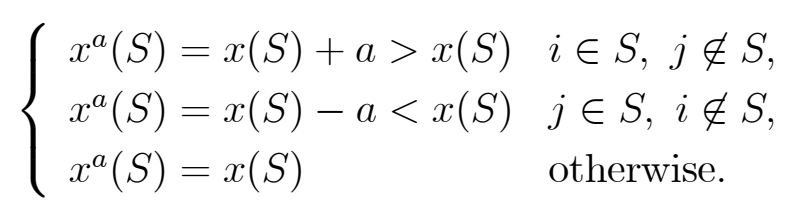

So, for every $S \in \Omega$ it is true that $x^{a}(S) \geq x(S)$ because $i$ is a superior of $j$ and thus there does not exist $S \in \Omega$ with $j \in S$ and $i \notin S$. We now have the following proposition.

Proposition 4.4 Let $(v, \Omega)$ be a monotone game on a union closed system and, for a vector $x$ and two players $i$ and $j$ such that $i$ is a superior of $j$, let $x^{a}$ be as defined in equation (4.1). Then

(i) if $x \in C(v, \Omega)$, then $x^{a} \in C(v, \Omega)$ for all $a \in\left(0, x_{j}\right]$.

(ii) if $x \in L C(v, \Omega)$ and $x_{i}<x_{j}$, then $x^{a} \in L C(v, \Omega)$ for all $a \in\left(0, \frac{1}{2}\left(x_{j}-x_{i}\right)\right]$.

Proof. To prove (i), recall from Proposition 4.1 that

$$
C(v, \Omega)=\left\{x \in X(v) x(S) \geq v(S) \text { for any } S \in \Omega \text { and } x_{j} \geq 0 \text { for any } j \in N\right\} .
$$

\footnotetext{
${ }^{4}$ There is some abuse of notation, actually $x^{a}$ also depends on $i$ and $j$.
} 
Clearly, for every $S \in \Omega$ we have that $x^{a}(S) \geq x(S) \geq v(S)$. Further, we have for every $k \neq j$ that $x_{k}^{a} \geq x_{k} \geq 0$ and that $x_{j}^{a}=x_{j}-a \geq 0$. Since $i$ is a superior of $j$ and thus $\{j\} \notin \Omega$, it follows that $x^{a} \in C(v, \Omega)$.

To prove (ii), notice that $x_{i}^{a} \leq x_{j}^{a}$ for all $a \in\left(0, \frac{1}{2}\left(x_{j}-x_{i}\right)\right]$. Suppose that $x^{a}$ is not in $L C(v, \Omega)$. Then there exists a coalition $S \subset N$ such that

$$
x^{a}(S)-r_{v, \Omega}(S)<e\left(r_{v, \Omega}\right) .
$$

Since $x \in L C(v, \Omega)$ we have that

$$
x(S)-r_{v, \Omega}(S) \geq e\left(r_{v, \Omega}\right) .
$$

Hence $x(S)>x^{a}(S)$, implying that $S$ contains $j$ but not $i$. Let $T=S \backslash\{j\}$ and $S^{\prime}=T \cup\{i\}$. Then

$$
x^{a}(S)-r_{v, \Omega}(S)=x^{a}(S)-v\left(\sigma_{\Omega}(T)\right)
$$

because $i \notin S$ and thus $j \notin \sigma_{\Omega}(S)$. Hence

$$
\begin{gathered}
x^{a}(S)-r_{v, \Omega}(S)=x^{a}(S)-v\left(\sigma_{\Omega}(T)\right) \geq x^{a}(T)+x_{j}^{a}-v\left(\sigma_{\Omega}(T \cup\{i\})\right) \geq \\
x^{a}(T)+x_{i}^{a}-v\left(\sigma_{\Omega}(T \cup\{i\})\right)=x^{a}\left(S^{\prime}\right)-r_{v, \Omega}\left(S^{\prime}\right),
\end{gathered}
$$

where the second inequality follows because $x_{i}^{a} \leq x_{j}^{a}$. So, with equation (4.2) it follows that

$$
x^{a}\left(S^{\prime}\right)-r_{v, \Omega}\left(S^{\prime}\right) \leq x^{a}(S)-r_{v, \Omega}(S)<e\left(r_{v, \Omega}\right)
$$

which contradicts that $x\left(S^{\prime}\right)-r_{v, \Omega}\left(S^{\prime}\right) \geq e\left(r_{v, \Omega}\right)$. The latter inequality must hold for $x \in L C(v, \Omega)$, since $x^{a}\left(S^{\prime}\right)>x\left(S^{\prime}\right)$ because $i \in S^{\prime}$ and $j \notin S^{\prime}$.

From Part (i) of Proposition 4.4 we obtain the following corollary, saying that when the core of the resticted game is not empty, there exist core stable payoff vectors that give zero payoff to every player $j$ that has a superior in $\Omega$.

Corollary 4.5 If $C\left(r_{v, \Omega}\right) \neq \emptyset$, then there exists $x \in C\left(r_{v, \Omega}\right)$ such that $x_{j}=0$ for every $j$ that has a superior.

The final proposition in this section states that for monotone games on a union closed system, a player gets at most the same payoff as its superior when applying the nucleolus to the restricted game. It should be noticed that when $v$ is monotone, $N u c_{j}(v, \Omega) \geq 0$ for all $j$, because $N u c(v, \Omega)$ is in the least core of $r_{v, \Omega}$ and thus also in $I\left(N, r_{v, \Omega}\right)$. 
Proposition 4.6 Let $(v, \Omega)$ be a monotone game on a union closed system. Then for every two players $i$ and $j$ such that $i$ is a superior of $j$ it holds that $N u c_{i}(v, \Omega) \geq N u c_{j}(v, \Omega)$.

Proof. Let $w \in \mathcal{G}^{N}$ be a game such that for every $S \subseteq N \backslash\{i, j\}$ it holds that $w(S \cup\{i\}) \geq$ $w(S \cup\{j\})$. Then we know from Peleg and Südholter (2003, Theorem 5.3.5) that $x_{i} \geq x_{j}$ for every $x$ in the prekernel of $w$. Since the nucleolus of a game is in the prekernel of a game, it is sufficient to show that for every $S \subseteq N \backslash\{i, j\}$ it holds that $r_{v, \Omega}(S \cup\{i\}) \geq r_{v, \Omega}(S \cup\{j\})$ when $i$ a superior of $j$. Indeed, in that case we have that

$$
r_{v, \Omega}(S \cup\{i\})=v\left(\sigma_{\Omega}(S \cup\{i\})\right) \geq v\left(\sigma_{\Omega}(S)\right)=v\left(\sigma_{\Omega}(S \cup\{j\})\right)=r_{v, \Omega}(S \cup\{j\}),
$$

where the second equality follows from the fact that $i \notin S$ and there does not exist a feasible set containing $j$ but not $i$.

In van den Brink et al. (2010) it is shown that also the Shapley value of the restricted game satisfies this property. ${ }^{5}$

\section{The prekernel of monotone games on union closed systems}

In this section we focus on the prekernel for games on union closed systems. Arin and Feltkamp (1997) proved that the kernel of a game $v \in \mathcal{G}^{N}$ consists of only one point (and coincides with the nucleolus), when the game is veto-rich and $I(v)$ is non-empty. When in the tuple $(v, \Omega)$ there exists a player $i \in N$ such that $i \in S$ for every $S \in \Omega$, then $i$ is a veto-player in the restricted game $r_{v, \Omega}$. When $v \in \mathcal{G}_{m}^{N}$ we have that $I\left(r_{v, \Omega}\right) \neq \emptyset$ and thus it follows from Arin and Feltkamp (1997) that the kernel of $r_{v, \Omega}$ has the nucleolus of $r_{v, \Omega}$ as its unique element. It is also well-known that for every game $(N, v)$ with $|N| \leq 3$, the intersection of the prekernel and the core consists of at most one point. In this section we generalize these results and give a sufficient condition to guarantee that the prekernel and the core of a monotone game on a union closed system have at most one point in common. Of course, when such a point exists, then it is the nucleolus of the restricted game. We first introduce some new notions.

Definition 5.1 For two players $i, j \in N, i \neq j$, player $i$ is a strong superior of player $j$ in $\Omega \in \mathcal{C}^{N}$ if $i$ is a superior of $j$ and $j$ is not a superior of $i$.

Definition 5.2 A player $i \in N$ is a free player in $\Omega \in \mathcal{C}^{N}$ if $i$ has no superiors; player $i \in N$ is a weakly free player in $\Omega \in \mathcal{C}^{N}$ if $i$ has no strong superiors.

\footnotetext{
${ }^{5}$ In this paper the Shapley value for games on union closed systems is introduced and characterized as the so-called union rule.
} 
Notice that a free player is also a weakly free player and that a weakly free player $i$ is a superior of $j$ when $j$ is a superior of $i$. For $\Omega \in \mathcal{C}^{N}$, we denote the set of weakly free players by

$$
W_{\Omega}=\{i \in N \mid i \text { is a weakly free player in } \Omega\} .
$$

The next proposition gives three properties of the set $W_{\Omega}$.

\section{Proposition 5.3}

1. For every player $j \notin W_{\Omega}$, there is a player $i \in W_{\Omega}$, such that $i$ is a strong superior of $j$.

2. When $j$ is a superior of a player $i \in W_{\Omega}$, then $i$ is a superior of $j$.

3. When $j$ is a superior of a player $i \in W_{\Omega}$, then $j \in W_{\Omega}$.

\section{Proof.}

1. Consider some player $i_{0} \in N$. If $i_{0}$ is not in $W_{\Omega}$, then $i_{0}$ has a strong superior, say $i_{1}$. Then, either $i_{1} \in W_{\Omega}$ and thus $i_{0}$ has a strong superior in $W_{\Omega}$, or not. In the latter case $i_{1}$ has a strong superior, say $i_{2}$. When $i_{2}$ is not in $W_{\Omega}$, it also has a strong superior. Continuing this we get a sequence of players $i_{0}, i_{1}, i_{2}, \ldots, i_{m}$ such that for $h=1, \ldots, m-1$, player $i_{h+1}$ is a strong superior of $i_{h}$ and thus $i_{h} \notin W_{\Omega}$ and either $i_{m} \in W_{\Omega}$ or $m \geq 2$ and $i_{m}=i_{k}$ for some $k=0, \ldots, m-2$. In the latter case, by Corollary 3.4 every pair $i_{j}, i_{\ell}$ with $j, \ell \in\{k, k+1, \ldots, m-1\}$ are superiors of each other, contradicting that $i_{h+1}$ is strong superior of $i_{h}, h=k, \ldots, m-1$. Hence every next player in the sequence is different from all preceding players. Since the number of players is finite, this case can not happen and thus within a finite number of steps some player $i_{m} \in W_{\Omega}$ is generated. By Corollary 3.4 $i_{m}$ is a superior of $i_{0}$. When $i_{0}$ is a superior of $i_{m}$, then again by Corollary 3.4 we have that $i_{0}$ is a superior of $i_{1}$, contradicting that $i_{1}$ is a strong superior of $i_{0}$. Hence $i_{m} \in W_{\Omega}$ and is a strong superior of $i_{0}$.

2. By definition, $i$ is a superior of $j$, since otherwise $j$ is a strong superior of $i$, which contradicts that $i \in W_{\Omega}$.

3. Suppose $j \notin W_{\Omega}$. Then by the first property, $j$ has a strong superior $k$ in $W_{\Omega}$. By Corollary 3.4 player $k$ is also a superior of $i$, and thus by property 2 we have that player $i$ is also a superior of $k$. However this implies that also $j$ is a superior of $k$, contradicting that $k$ is a strong superior of $j$.

The first property of Proposition 5.3 yields the following corollary.

Corollary 5.4 For every $\Omega \in \mathcal{C}^{N}, W_{\Omega} \neq \emptyset$.

Next, for $i \in W_{\Omega}$, define $T_{\Omega}(i)=\{j \in N \mid j=i$ or $j$ is a superior of $i\}$ and let $\mathcal{T}_{\Omega}$ be the collection of sets defined by

$$
\mathcal{T}_{\Omega}=\left\{T_{\Omega}(i) \mid i \in W_{\Omega}\right\} .
$$


Notice that for every $j \in T_{\Omega}(i) \backslash\{i\}$, also $i$ is a superior of $j$, because $i \in W_{\Omega}$, and thus $T_{\Omega}(i) \subseteq S_{i}^{\Omega}=\{j \in N \mid j=i$ or $i$ is a superior of $j\}$. The next proposition describes the set $W_{\Omega}$.

Proposition 5.5 The collection $\mathcal{T}_{\Omega}$ is a partition of the set $W_{\Omega}$.

Proof. First, by Property 3 of Proposition 5.3 we have that $j \in W_{\Omega}$ when $j \in T_{\Omega}(i)$ for some $i \in W_{\Omega}$ and thus $T_{\Omega}(i) \subseteq W_{\Omega}$. Next, let $R \subseteq W_{\Omega} \times W_{\Omega}$ be the binary relation on $W_{\Omega}$ defined by $(j, i) \in R$ if and only if $j \in T_{\Omega}(i)$. It is sufficient to show that this relation is an equivalence relation on $W_{\Omega}$, i.e. the relation is reflexive, symmetric and transitive. First, by definition $(i, i) \in R$ for all $i \in W_{\Omega}$, so $R$ is reflexive. Second, for $j \neq i$, when $(j, i) \in R$, then $j$ is a superior of $i$. By Property 2 of Proposition 5.3 then also $i$ is a superior of $j$ and thus $(i, j) \in R$, showing that $R$ is symmetric. Third, when $(k, j) \in R$ and $(j, i) \in R$, then $k$ is a superior of $j$ and $j$ of $i$ and thus, by Corollary 3.4, also $k$ is a superior of $i$. Hence, $(k, i) \in R$ and thus $R$ is transitive. Since $R$ is an equivalence relation, it follows that the sets $T_{\Omega}(i), i \in W_{\Omega}$, are equivalence classes of $W_{\Omega}$ and thus the collection $\mathcal{T}_{\Omega}$ partitions $W_{\Omega}$.

Proposition 5.5 implies that $j \in T_{\Omega}(i)$ if and only if $i \in T_{\Omega}(j)$. When, for two different agents $i, j \in W_{\Omega}, i$ is not a superior of $j$, then $T_{\Omega}(i)$ and $T_{\Omega}(j)$ are two different equivalence classes.

Proposition 5.6 Let $\Omega$ be a union closed system. When $j \in W_{\Omega}$ is a superior of $i \in N$ then every $k \in T_{\Omega}(j)$ is a superior of $i$.

Proof. For $i \in W_{\Omega}$ the proposition follows from Proposition 5.5, because $T_{\Omega}(i)=T_{\Omega}(j)$ when $j$ is a superior of $i$. Let $i \notin W_{\Omega}$ and $j \in W_{\Omega}$ be a superior of $i$. Then every $k \neq j$ in $T_{\Omega}(j)$ is a superior of $j$ and thus a superior of $i$ by Corollary 3.4.

Proposition 5.7 Let $(v, \Omega)$ be a game on a union closed system. When $\mathcal{T}_{\Omega}$ consists of only one set, then every player in $W_{\Omega}$ is a veto-player in the restricted game $r_{v, \Omega}$.

Proof. First, when $\mathcal{T}_{\Omega}$ consists of only one set, say $T$, then, by Proposition $5.5, T=W_{\Omega}$. So, $T_{\Omega}(i)=W_{\Omega}$ for every $i \in W_{\Omega}$ and thus by definition of $T_{\Omega}(i)$ and Proposition 5.6 every player $k \in W_{\Omega}$ is a superior to every other $i$ in $W_{\Omega}$. Moreover, by Property 1 of Proposition 5.3 every player not in $W_{\Omega}$ has a player $i$ in $W_{\Omega}$ as its superior, and thus, again by Proposition 5.6, every player in $W_{\Omega}$ is a superior of every player not in $W_{\Omega}$. So, every player in $W_{\Omega}$ is a superior of every other player in $N$, so that every $S \in \Omega$ contains all players in $W_{\Omega}$. 
Notice that $T_{\Omega}(i)=\{i\}$ when $i$ is a free player. So, every free player $i$ gives a single element equivalence class $T_{\Omega}(i)=\{i\}$ in the partition $\mathcal{T}_{\Omega}$ of $W_{\Omega}$. When there is a free player $i$ and $\mathcal{T}_{\Omega}$ consists of only one set, then $W_{\Omega}=\{i\}$. In the sequel we call the number of sets in $\mathcal{T}_{\Omega}$ the weakly free player cardinality of $\Omega$. Since by Corollary 5.4 the set of weakly free players is not-empty, this cardinality is at least one. It follows from Proposition 5.7 that $r_{v, \Omega}$ is a veto-rich game when this cardinality is equal to one. Then the next corollary follows from Arin and Feltkamp (1997).

Corollary 5.8 Let $(v, \Omega)$ be a game on a union closed system. Then the kernel $K\left(r_{v, \Omega}\right)$ contains the nucleolus $N u c\left(r_{v, \Omega}\right)$ as its unique element when the weakly free player cardinality is one.

To generalize this, we use the famous theorem of Kohlberg (1971) giving a sufficient and necessary condition for a payoff vector to be in the prenucleolus of a game. For game $v \in \mathcal{G}^{N}$, a payoff vector $x \in \mathbb{R}^{n}$ and real number $\alpha$, let $\mathcal{B}(\alpha, x)$ be the collection of coalitions given by $\mathcal{B}(\alpha, x)=\{S \in N \mid e(S, x) \geq \alpha\}$.

Theorem 5.9 (Kohlberg, 1971) For game $v \in \mathcal{G}^{N}$, a payoff vector $x$ is in $P N(v)$ if and only if for any real number $\alpha$ the collection of coalitions $\mathcal{B}(\alpha, x)$ is either balanced or empty.

In Katsev and Yanovskaya (2010) an analogue of this theorem for the prekernel is proved in terms of 2-balancedness. We first give the notion of $k$-balancedness for $2 \leq k \leq n$.

Definition 5.10 A collection $\mathcal{S}$ of coalitions $S \in 2^{N}$ is $k$-balanced if for every coalition $K \subseteq N$ with $|K|=k$ the collection $\mathcal{S}_{K}=\left\{S^{\prime} \subset K \mid S^{\prime}=S \cap K, S \in \mathcal{S}\right\}$ is balanced on $K$.

Theorem 5.11 (Katsev and Yanovskaya, 2010) For $v \in \mathcal{G}^{N}$, a payoff vector $x$ is in $P K(v)$ if and only if for any real number $\alpha$ the collection of coalitions $\mathcal{B}(\alpha, x)$ is either 2-balanced or empty.

Recall from the standard definition of balancedness that when a collection $\mathcal{S}_{K}$ is balanced on $K$, then there exist strictly positive weights $\lambda_{T}, T \in \mathcal{S}_{K}$, such that for every $i \in K$ the total weight of the sets $T \in \mathcal{S}_{K}$ that contain $i$ is equal to one. From this the following corollary follows immediately.

Corollary 5.12 Let $K=\{i, j\} \subseteq N$ be a two-player coalition and $\mathcal{S}$ be a collection of coalitions $S \in 2^{N}$ such that $\mathcal{S}_{K}$ is balanced on $K$. When $\mathcal{S}$ contains a set $T$ such that $i \in T$ and $j \notin T$, then $\mathcal{S}$ contains a coalition $T^{\prime}$ such that $j \in T^{\prime}$ and $i \notin T^{\prime}$. 
Also notice that a $k$-balanced collection $\mathcal{S}$ is balanced when $k=n$. Moreover it should be noticed that when $|N|=3$, any 2-balanced collection is also balanced. The next lemma generalizes this fact and will be used to prove the main result of this section.

Lemma 5.13 For a union closed system $\Omega$ with weakly free player cardinality of at most three, let $\mathcal{B} \subset 2^{N}$ be a 2-balanced collection that only contains feasible sets in $\Omega$ and singletons. Then $\mathcal{B}$ is balanced.

Proof. Let $c \in\{1,2,3\}$ be the weakly free player cardinality of $\Omega$. Without loss of generality, let the players be numbered in such way that $W_{\Omega} \supset\{1, \ldots, c\}$ and that $T_{\Omega}(k)$, $k=1, \ldots, c$, are the equivalence classes of $\mathcal{T}_{\Omega}$. By property 2 of Proposition 5.3, every player $j \neq k$ in $T_{\Omega}(k)$ has player $k$ as its superior. Also, by property 1 of Proposition 5.3 and by Proposition 5.6, every player $j \in N \backslash W_{\Omega}$ has at least one of the players $k, k \in W_{\Omega}$ as one of its superiors. For $k \in W_{\Omega}$, suppose that there exists $j$ in the set

$$
S_{k}^{\Omega} \backslash\{k\}=\{i \in N \mid k \text { is a superior of } i\}
$$

such that there is some $T$ in $\mathcal{B}$ containing $k$, but not $j$. Take $K=\{k, j\}$. By the 2balancedness of $\mathcal{B}$ the collection $\{S \cap K \mid S \in \mathcal{B}\}$ is balanced on $K$. So, by Corollary 5.12 there exists a set $T^{\prime} \in \mathcal{B}$ such that $j \in T^{\prime}$ and $k \notin T^{\prime}$. Since $\mathcal{B}$ only contains feasible sets and singletons, and $k$ is a superior of $j$, it follows that $T^{\prime}=\{j\}$. Let

$$
S_{k}=\cap_{\{S \in \mathcal{B} \mid k \in S\}} S, \quad k \in W_{\Omega} .
$$

From above it follows that $\{j\} \in \mathcal{B}$ for every $j \notin \cup_{k \in W_{\Omega}} S_{k}$. Now, let

$$
\mathcal{B}^{\prime}=\left\{U \in \mathcal{B} \mid U \cap W_{\Omega} \neq \emptyset\right\}
$$

and consider the collection of subsets of $W_{\Omega}$ given by

$$
\mathcal{B}^{\prime \prime}=\left\{W_{\Omega} \cap U \mid U \in \mathcal{B}^{\prime}\right\} .
$$

This is a balanced collection on $W_{\Omega}$. This is trivial when $c=1$ and follows by the 2balancedness of $\mathcal{B}$ when $c=2$. When $c=3$ this follows from the fact that every 2-balanced collection on a three player set is balanced. So, for the sets $U \in \mathcal{B}^{\prime}$ there are weights, say $\lambda_{U}^{\mathcal{B}}$, such that

$$
\sum_{\left\{U \in \mathcal{B}^{\prime} \mid k \in U\right\}} \lambda_{U}^{\mathcal{B}}=1, \quad k \in W_{\Omega} .
$$

Since every feasible set has a nonempty intersection with $W_{\Omega}$, this yields weight $\lambda_{U}^{\mathcal{B}}>0$ for every feasible set $U \in \mathcal{B}$. Moreover,

$$
\sum_{\left\{U \in \mathcal{B}^{\prime} \mid j \in U\right\}} \lambda_{U}^{\mathcal{B}}=1, \quad \text { for every } j \in \cup_{k=1, \ldots, c} S_{k},
$$


since if $j \in S_{k}$ for some $k=1, \ldots, c$, then the collection of sets from $\mathcal{B}^{\prime}$ containing $j$ coincides with the collection of sets from $\mathcal{B}$ ' containing $k$. Finally, consider some $j \in$ $N \backslash\left(\cup_{k=1, \ldots, c} S_{k}\right)$. Recall that such a player $j$ has at least one of the players from the set $W_{\Omega}$ as one of its superiors, say player $k$. So, when $j$ is contained in some set $U \in \mathcal{B}^{\prime}$, then also $k \in U$. Moreover, there exists at least one $U \in \mathcal{B}^{\prime}$ containing $k$ and not $j$, otherwise $j \in S_{k}$. Therefore,

$$
\sum_{\left\{U \in \mathcal{B}^{\prime} \mid j \in U\right\}} \lambda_{U}^{\mathcal{B}}<1 \text { for every } j \in N \backslash\left(\cup_{k=1, \ldots, c} S_{k}\right),
$$

i.e., the total weight of the feasible sets containing such a player $j$ is less than one. However, for every such $j$ we also have that the singleton $\{j\} \in \mathcal{B}$. This yields weight $\lambda_{\{j\}}^{\mathcal{B}}=$ $1-\sum_{\left\{U \in \mathcal{B}^{\prime} \mid j \in U\right\}} \lambda_{U}^{\mathcal{B}}$ for every singleton set $\{j\} \in \mathcal{B}, j \in N \backslash\left(\cup_{k=1, \ldots, c} S_{k}\right)$. Since for every $j \in \cup_{k=1, \ldots, c} S_{k}$, every set in $\mathcal{B}$ containing $j$ also contains one of the players from $\{1, \ldots, c\}$, there are no other singletons in $\mathcal{B}$. So, we have determined weights for all sets in $\mathcal{B}$ satisfying that

$$
\sum_{\{S \in \mathcal{B} \mid j \in S\}} \lambda_{S}^{\mathcal{B}}=1 \text {, for every } j \in N
$$

and thus $\mathcal{B}$ is balanced.

We are now ready to formulate the main result of this section.

Theorem 5.14 Let $(v, \Omega)$ be a monotone game on a union closed system. Then the intersection of $P K(v, \Omega)$ and $C(v, \Omega)$ consists of at most one point if the weakly free player cardinality of $\Omega$ is at most equal to three.

Proof. Clearly, the statement of the theorem is true when $C(v, \Omega)=\emptyset$. So, we only consider the case that $C(v, \Omega) \neq \emptyset$. Then $P N(v, \Omega)=N u c(v, \Omega)$ and lies in the core. Suppose there is a payoff vector $y \in P K(v, \Omega) \cap C(v, \Omega)$ with $y \neq x=N u c(v, \Omega)$. Since $y \neq P N(v, \Omega)$, according to Kohlberg's theorem there is some $\alpha$ for which $\mathcal{B}(\alpha, y)$ is not balanced. Since $x=P N(v, \Omega)$, also according to Kohlberg's theorem we have that $\mathcal{B}(\alpha, x)$ is balanced and thus $\mathcal{B}(\alpha, x) \neq \mathcal{B}(\alpha, y)$. Since for $\alpha$ big enough we have that $\mathcal{B}(\alpha, x)=\mathcal{B}(\alpha, y)=\emptyset$, there exists some value $\alpha$ with the properties that

(i) $\mathcal{B}(\alpha, x) \neq \mathcal{B}(\alpha, y)$ and

(ii) for every $\beta>\alpha$ it is true that either $\mathcal{B}(\beta, x)=\mathcal{B}(\beta, y)$ or both $\mathcal{B}(\alpha, x)=\mathcal{B}(\beta, x)$ and $\mathcal{B}(\alpha, y)=\mathcal{B}(\beta, y)$.

For a coalition $S$ and payoff vector $x$, let $e(S, x)=r_{v, \Omega}(S)-x(S)=v\left(\sigma_{\Omega}(S)\right)-x(S)$ be the excess of coalition $S$ at $x$ in the restricted game $r_{v, \Omega}$ and let $\alpha^{*}$ be a value satisfying the two properties (i) and (ii). Now, suppose that there exists $S \in \mathcal{B}\left(\alpha^{*}, x\right)$ such that 
$e(S, x)<e(S, y)$. Then, for $\beta=e(S, y)>e(S, x) \geq \alpha^{*}$, we have that $S \in \mathcal{B}(\beta, y)$ and $S \notin \mathcal{B}(\beta, x)$. So, $\mathcal{B}(\beta, x) \neq \mathcal{B}(\beta, y)$ and $\mathcal{B}\left(\alpha^{*}, x\right) \neq \mathcal{B}(\beta, x)$, which contradicts that property (ii) holds for $\alpha^{*}$. Hence

$$
e(S, x) \geq e(S, y) \text { for every } S \in \mathcal{B}\left(\alpha^{*}, x\right)
$$

Further, for $S \in \mathcal{B}\left(\alpha^{*}, x\right)$, let $\lambda_{S}$ be the weight of $S$ in the balanced system of collection $\mathcal{B}\left(\alpha^{*}, x\right)$. Since both $x$ and $y$ are efficient, it follows that

$$
\sum_{\left\{S \mid S \in \mathcal{B}\left(\alpha^{*}, x\right)\right\}} \lambda_{S} e(S, x)=\sum_{\left\{S \mid S \in \mathcal{B}\left(\alpha^{*}, x\right)\right\}} \lambda_{S}\left(r_{v, \Omega}(S)-x(S)\right) .
$$

Since $x$ is efficient and by balancedness we have that $\sum_{\left\{S \in \mathcal{B}\left(\alpha^{*}, x\right) \mid i \in S\right\}} \lambda_{S}=1$ for every $i \in N$, it follows that

$$
\sum_{S \in \mathcal{B}\left(\alpha^{*}, x\right)} \lambda_{S} x(S)=\sum_{S \in \mathcal{B}\left(\alpha^{*}, x\right)} \lambda_{S} \sum_{i \in S} x_{i}=\sum_{i \in N} x_{i} \sum_{\left\{S \in \mathcal{B}\left(\alpha^{*}, x\right) \mid i \in S\right\}} \lambda_{S}=\sum_{i \in N} x_{i}=r_{v, \Omega}(N),
$$

and thus

$$
\begin{aligned}
\sum_{\left\{S \mid S \in \mathcal{B}\left(\alpha^{*}, x\right)\right\}} \lambda_{S} e(S, x) & =\sum_{\left\{S \mid S \in \mathcal{B}\left(\alpha^{*}, x\right)\right\}} \lambda_{S}\left(r_{v, \Omega}(S)-x(S)\right) . \\
& =\sum_{\left\{S \mid S \in \mathcal{B}\left(\alpha^{*}, x\right)\right\}} \lambda_{S} r_{v, \Omega}(S)-r_{v, \Omega}(N) .
\end{aligned}
$$

Analogously

$$
\sum_{\left\{S \mid S \in \mathcal{B}\left(\alpha^{*}, x\right)\right\}} \lambda_{S} e(S, y)=\sum_{\left\{S \mid S \in \mathcal{B}\left(\alpha^{*}, x\right)\right\}} \lambda_{S} r_{v, \Omega}(S)-r_{v, \Omega}(N) .
$$

So,

$$
\sum_{\left\{S \mid S \in \mathcal{B}\left(\alpha^{*}, x\right)\right\}} \lambda_{S} e(S, x)=\sum_{\left\{S \mid S \in \mathcal{B}\left(\alpha^{*}, x\right)\right\}} \lambda_{S} e(S, y)
$$

With inequalities (5.3) this implies $e(S, y)=e(S, x)$ for every $S \in \mathcal{B}\left(\alpha^{*}, x\right)$ and thus $\mathcal{B}\left(\alpha^{*}, x\right) \subseteq \mathcal{B}\left(\alpha^{*}, y\right)$.

Now, suppose that also the collection $B\left(\alpha^{*}, y\right)$ is balanced. Then by the same reasoning as above we obtain that $e(S, x)=e(S, y)$ for every $S \in \mathcal{B}\left(\alpha^{*}, y\right)$ and thus also $\mathcal{B}\left(\alpha^{*}, y\right) \subseteq \mathcal{B}\left(\alpha^{*}, x\right)$, which contradicts that $\mathcal{B}\left(\alpha^{*}, x\right) \neq \mathcal{B}\left(\alpha^{*}, y\right)$. Hence $B\left(\alpha^{*}, y\right)$ is not balanced.

On the other hand, by Theorem 5.11 we have that $\mathcal{B}\left(\alpha^{*}, y\right)$ is 2-balanced, since $y \in P K(v, \Omega)$. So, $\mathcal{B}\left(\alpha^{*}, y\right)$ is 2-balanced, but not balanced. Then, according to Lemma $5.13, \mathcal{B}\left(\alpha^{*}, y\right)$ contains a non-feasible coalition $S$ with $|S|>1$. By definition of $\sigma_{\Omega}(S)$ and 
$\Omega$ being union closed, we have that $r_{v, \Omega}(T)=0$ for every $T \subseteq S \backslash \sigma_{\Omega}(S)$. Then for every $i \in S \backslash \sigma_{\Omega}(S)$ it follows that

$$
\begin{gathered}
e(S, y)=r_{v, \Omega}(S)-y(S)=r_{v, \Omega}\left(\sigma_{\Omega}(S)\right)-\sum_{j \in \sigma_{\Omega}(S)} y_{j}-\sum_{h \in S \backslash \sigma_{\Omega}(S)} y_{h} \leq \\
e\left(\sigma_{\Omega}(S), y\right)-y_{i}=e\left(\sigma_{\Omega}(S), y\right)+e(\{i\}, y),
\end{gathered}
$$

because $y \in C\left(r_{v, \Omega}\right)$ and thus $y_{h} \geq r_{v, \Omega}(\{h\})=0$ for all $h \in S \backslash \sigma_{\Omega}(S)$. Since both $e\left(\sigma_{\Omega}(S), y\right) \leq 0$ and $e(\{i\}, y) \leq 0$ (again because $y \in C\left(r_{v, \Omega}\right)$ ), it follows that

$$
\left.e(S, y) \leq e\left(\sigma_{\Omega}(S), y\right) \text { and } e(S, y) \leq e(\{i\}), y\right)
$$

Hence both $\sigma(S) \in \mathcal{B}\left(\alpha^{*}, y\right)$ and $\{i\} \in \mathcal{B}\left(\alpha^{*}, y\right)$ for every $i \in S \backslash \sigma_{\Omega}(S)$. However, then also the collection $\mathcal{B}\left(\alpha^{*}, y\right) \backslash\{S\}$ is 2-balanced and not balanced. Let $N F=\{T \in$ $\mathcal{B}\left(\alpha^{*}, y\right) \mid T$ is non-feasible and $\left.|T|>1\right\}$. Repeating the reasoning above for every $T \in N F$ it follows that $\mathcal{B}^{\prime}=\mathcal{B}\left(\alpha^{*}, y\right) \backslash N F$ is 2-balanced and not balanced. However, since $\mathcal{B}^{\prime}$ only consists of feasible sets and singletons, this contradicts Lemma 5.13. So, there is no $y \in P K(v, \Omega) \cap C(v, \Omega)$ with $y \neq x=N u c(v, \Omega)$.

\section{References}

Algaba, E., J.M. Bilbao, R. van den Brink, And A. Jiménez-Losada (2003), Axiomatizations of the Shapley value for cooperative games on antimatroids, Mathematical Methods of Operations Research, 57, 49-65.

Algaba, E., J.M. Bilbao, R. van den Brink, And A. Jiménez-Losada (2004), Cooperative games on antimatroids, Discrete Mathematics, 282, 1-15.

AlGABA, E., J. M. Bilbao, And M. Slikker (2010), A value for games restricted by augmenting systems, SIAM Journal on Discrete Mathematics, 17, 122-133.

Arin, Feltkamp (1997), The nucleolus and kernel of veto-rich transferable utility games, International Journal of Game Theory, 26, 61-73.

Bilbao, J.M. (2000), Cooperative Games on Combinatorial Structures, Kluwer Academic Publishers.

Bilbao, J.M. (2003), Cooperative Games under augmenting systems, SIAM Journal on Discrete Mathematics, 24, 992-1010.

Bilbao, J.M., And M. Ordóñez (2009), The core and the Weber set of games on augmenting systems, Discrete Applied Mathematics, 158, 180-188. 
Bondareva (1962), The theory of the core in an $n$-person game, Vestnik Leningrad. Univ., 13, 141-142 (in Russian).

Brink, R. VAn Den (1997), An Axiomatization of the Disjunctive Permission Value for Games with a Permission Structure, International Journal of Game Theory, 26, 2743.

Brink R. van Den, And R.P. Gilles (1996), Axiomatizations of the Conjunctive Permission Value for Games with Permission Structures, Games and Economic Behavior, 12, 113-126.

Brink, R. van den, I. Katsev And G. van Der LaAn (2011), Axiomatizations of Two Types of Shapley Values for Games on Union Closed Systems, forthcoming in Economic Theory.

Dilworth, R. P. (1940) Lattices with Unique Irreducible Decompositions, Annals of Mathematics, 41, 771-777.

Edelman, P. H., And Jamison, R. E. (1985) The Theory of Convex Geometries, Geometrica Dedicata, 19, 247-270.

Einy, Holzman, Monderer (1999), On the least core and the Mas-Colell bargaining set, Games and Economic Behavior, 28, 181-188.

Gilles, R. P., And Owen, G. (1994), Cooperative games and disjunctive permission structures, Department of Economics, Virginia Polytechnic Institute and State University, Blacksburg, Virginia.

Gilles R.P., G. Owen, And R. van den Brink (1992), Games with Permission Structures: The Conjunctive Approach, International Journal of Game Theory, 20, 277293.

Harsanyi, J.C. (1959), A Bargaining Model for Cooperative n-Person Games, In Contributions to the Theory of Games IV (eds. Tucker A.W., and R.D. Luce), Princeton UP, Princeton pp 325-355.

Katsev, I. And Yanovskaya, E. (2010), Between the prekernel and the prenucleolus, Working paper, Russian Academy of Sciences (RAS), St. Petersburg Institute for Economics and Mathematics,

Kohlberg (1971), On the nucleolus of a characteristic function game, SIAM Journal of Applied Mathematics, 20, 62-66. 
Maschler M., Peleg, B., And L.S. Shapley (1979), Geometric properties of the kernel, nucleolus, and related solution concepts, Mathematics of Operations Research, 4, 303Ü338,

Myerson, R.B. (1977), Graphs and Cooperation in Games, Mathematics of Operations Research, 2, 225-229.

Peleg AND SÜDholter (2003), Introduction to the theory of cooperative games, Boston, Dordrecht, London: Kluwer Acad. Publ.

Shapley, L.S. (1953) A Value for n-Person Games, In Annals of Mathematics Studies 28 (Contributions to the Theory of Games Vol.2) (eds. Kuhn H.W., and A.W. Tucker), Princeton UP, Princeton pp 307-317.

Shapley, L.S. (1967), On balanced sets and cores, Naval Research Logistics Quarterly, 14, 453-460. 